\title{
La Chine, l'Inde et le nouveau régionalisme asiatique
}

Ajustements et repositionnements

China, India and the new Regionalism in East Asia: Current Adjustments and Repositioning in Asia.

\section{Isabelle Saint-Mézard}

\section{OpenEdition}

\section{Journals}

Édition électronique

URL : http://journals.openedition.org/transcontinentales/336

DOI : 10.4000/transcontinentales.336

ISBN : 978-2-8218-1410-3

ISSN : 1775-397X

\section{Éditeur}

Editions de la maison des sciences de l'homme

Édition imprimée

Date de publication : 31 décembre 2005

Pagination : 125-140

ISBN : 2200-92168-3

ISSN : 1950-1684

Référence électronique

Isabelle Saint-Mézard, « La Chine, I'Inde et le nouveau régionalisme asiatique », Transcontinentales [En ligne], 1 | 2005, document 9, mis en ligne le 03 février 2011, consulté le 10 décembre 2020. URL : http://journals.openedition.org/transcontinentales/336 ; DOI : https://doi.org/10.4000/ transcontinentales.336

Ce document a été généré automatiquement le 10 décembre 2020.

Tous droits réservés 


\title{
La Chine, l'Inde et le nouveau régionalisme asiatique
}

\author{
Ajustements et repositionnements \\ China, India and the new Regionalism in East Asia: Current Adjustments and \\ Repositioning in Asia.
}

Isabelle Saint-Mézard

1 À bien des égards, la comparaison entre les positions respectives de l'Inde et de la Chine sur la scène régionale asiatique révèle des parallèles intéressants, tout en apportant un éclairage singulier sur l'évolution de leurs relations bilatérales. Sur l'histoire longue, les deux pays figurent comme deux grandes matrices culturelles dont l'influence, tant politique, que philosophique et économique, s'est manifestée sur une grande partie de l'Asie au fil des siècles. L'histoire récente, en particulier la période de la guerre froide, évoque cependant un rapport houleux à leur voisinage, notamment en Asie du Sud-Est. Dans le cas de l'Inde, il s'agit surtout d'une distanciation des liens traditionnels avec les voisins d'Asie orientale, le pays se repliant sur l'Asie du Sud tout en embrassant l'amitié soviétique ; la Chine a quant à elle, et plus gravement, laissé le souvenir durable de sa politique de soutien aux guérillas communistes en Asie du Sud-Est, d'où les réflexes de suspicion qu'elle continue de provoquer chez certains de ses voisins.

2 Mais depuis la fin des années 1980 et tout au long des années 1990, la Chine comme l'Inde ont multiplié les efforts pour se rapprocher de leurs voisins d'Asie du Sud-Est, au moment même où ceux-ci se sont mis à relancer leurs initiatives de coopération régionale. New Delhi et Pékin ont par exemple accédé au statut de partenaire de dialogue de l'ASEAN la même année, en 1996, et sont parallèlement devenues membres du forum régional de l'ASEAN (ARF), alors la seule organisation régionale à s'intéresser aux questions de sécurité en Asie. Ayant respectivement entrepris de réformer leur économie à la fin des années 1970 et au tout début des années 1990, Chinois et Indiens ont aussi considérablement accru les liens commerciaux et financiers avec leurs dynamiques voisins d'Asie du Sud-Est et du Nord-Est. Au reste, dans l'Asie de l'après crise financière, les deux pays se profilent de plus en plus comme les grands moteurs 
économiques de la région. De façon plus générale, il semble qu'après avoir été pendant des décennies obnubilées par leur rapport à l'occident, les élites dirigeantes chinoises et indiennes redécouvrent et réinvestissent leur environnement régional, avec une sensibilité asiatique de plus en plus prononcée.

3 En dépit de ces traits communs, la Chine paraît aujourd'hui dans une position privilégiée sur la scène régionale asiatique. Plus précisément, la Chine est devenue un acteur prédominant dans les développements qui caractérisent le régionalisme asiatique depuis la crise financière de 1997. L'Inde semble un peu plus en retrait, ou à tout le moins à la marge de ces mêmes développements régionaux, cela en dépit d'une politique de "Regard vers l'Est» (Look East policy) pratiquée avec une certaine constance depuis le début des années 1990. Certains échecs au cours de cette décennie ont, de fait, sanctionné un statut de "partenaire de second rang» du régionalisme asiatique. En dépit de ses demandes réitérées, l'Inde n'est par exemple parvenue à se faire admettre ni au sein du forum de coopération économique de l'Asie Pacifique (APEC), ni au sein du sommet Asie-Europe (ASEM) ${ }^{1}$. Mais New Delhi ne se satisfait guère de cette position périphérique. Et à la faveur de la nouvelle vague de régionalisme qui touche l'Asie orientale depuis la fin des années 1990, l'Inde tend de plus en plus à emboîter le pas des initiatives chinoises, en matière économique comme diplomatique.

Cet article a précisément pour objet de mettre en relief cette émulation entre Indiens et Chinois pour être partie prenante des nouvelles dynamiques d'intégration en Asie orientale. Ce faisant, il examine comment les deux puissances se positionnent l'une par rapport à l'autre dans cette course à l'intégration régionale. Nous ne saurions en effet ignorer que ces deux voisins ont eu des relations chaotiques par le passé et que le rapprochement bilatéral en cours ne les empêche en rien de cultiver une vision ambitieuse de leur rôle historique en Asie. Dès lors, on peut s'interroger sur ce qui anime l'empressement de l'Inde et de la Chine à être en première ligne des développements régionaux : rivalité larvée, émulation pacifique ou encore, incitation à améliorer leurs propres relations bilatérales? Pour jauger la validité de ces diverses hypothèses, nous nous proposons d'analyser les regards croisés que les acteurs indiens et chinois portent sur les efforts de l'autre partie pour s'intégrer à la régionalisation asiatique.

\section{Initiatives indiennes et chinoises face au renouveau du régionalisme asiatique}

5 L'activisme de l'Inde et de la Chine en Asie orientale peut se comparer en trois domaines particuliers, qui touchent successivement à leur participation aux mécanismes institutionnels de coopération régionale, à leurs efforts pour conclure des accords de libre-échange et à leur volonté de contribuer à la sécurité de la région.

\section{Participer aux nouveaux mécanismes de coopération régionale : ASEAN+3 et ASEAN+Inde}

6 La crise financière de 1997 a sans nul doute accru le sentiment d'interdépendance économique au sein de la région. Le mécontentement général à l'égard des interventions du Fond monétaire international aidant, les pays d'Asie orientale ont entrepris de renforcer les mécanismes de coordination régionaux, notamment dans le 
secteur financier, afin de gérer de façon collective et autonome toute nouvelle crise éventuelle. Ce faisant, un nouveau concept régional a émergé avec la structuration progressive de l'ASEAN+3, soit les dix membres de l'ASEAN et leurs trois principaux partenaires en Asie du Nord-Est, le Japon, la Corée du Sud et la Chine²(voir carte p. 136). Cette dernière a justement vu sa position se renforcer encore à l'occasion de la crise de 1997, grâce à une habile politique d'aide aux économies en difficulté et d'appui marqué à l'initiative d'ASEAN+3. Ainsi, lorsqu'en décembre 1997 s'est tenue la première réunion de ce qui allait bientôt correspondre au format ASEAN+3, les dirigeants chinois se sont distingués par leur claire posture de soutien à l'égard de ce nouveau mécanisme de coopération régionale promu par une ASEAN alors à la recherche d'un nouveau souffle.

7 En quelques années, l'ASEAN+3 s'est imposée comme la force motrice du nouveau régionalisme asiatique. Quant à la Chine, elle ne s'est pas seulement contentée d'encourager l'ASEAN +3 , elle en est devenue l'un des membres les plus actifs, prenant ainsi souvent de court les deux autres partenaires nord-est asiatiques, la Corée du Sud et surtout le Japon ${ }^{3}$. En l'occurrence, la Chine ne perd pas de vue ses propres objectifs et intérêts diplomatiques: le soutien apporté à l'ASEAN+3 et au lointain projet d'une communauté d'Asie orientale participe d'une politique de restauration de la confiance avec l'ensemble du voisinage, Pékin ayant clairement en vue de contrecarrer la thèse de la «menace chinoise" qui continue de hanter une bonne partie de la région ${ }^{4}$. La signature en octobre 2003 d'une déclaration conjointe sino-ASEAN de "partenariat stratégique pour la paix et la prospérité » procède clairement de cette vaste entreprise de resocialisation régionale menée à marche forcée en Asie orientale. Du point de vue de Pékin, la formalisation de cet ambitieux partenariat avec l'ASEAN constitue l'assise même du processus plus vaste d'intégration régionale en Asie orientale.

Largement épargnée par la crise financière de 1997, l'Inde s'est trouvée dans une posture paradoxale : certes, elle faisait figure d'économie parmi les plus stables en Asie, mais elle était encore une fois laissée à la marge des nouvelles dynamiques d'intégration régionale à l'œuvre dans son voisinage. Comprenant ces enjeux, New Delhi a, dès novembre 2000, demandé de façon informelle à prendre part au mécanisme ASEAN+3. Mais les pays d'Asie du Sud-Est se sont montrés peu favorables à l'idée d'une expansion de leur initiative en "ASEAN+4 » : ils ont à défaut proposé un mécanisme spécifique, l'ASEAN+Inde, dont la première réunion s'est tenue fin 2002. Les mécanismes parallèles - mais clairement distincts - d'ASEAN+3 et d'ASEAN+Inde résument finalement bien la position respective de la Chine et de l'Inde au sein du nouveau régionalisme asiatique. La première est non seulement partie prenante des développements régionaux, mais elle est progressivement devenue un élément central du projet collectif de communauté d'Asie orientale; la seconde persévère dans un statut de partenaire alternatif (comprendre hors Asie orientale), pourtant de plus en plus influent et indispensable du point de vue même de l'ASEAN. Au reste, l'ASEAN a clairement signifié son désir de renforcer les liens avec l'Inde en concluant, en novembre 2004, un "partenariat pour la paix, le progrès et une prospérité partagée ", qui d'une certaine façon fait contrepoint au partenariat stratégique signé avec la Chine en $2003^{5}$. 


\section{Promouvoir des accords de libre-échange et soutenir la croissance régionale}

L'émulation entre Indiens et Chinois pour être partie prenante de l'intégration régionale asiatique se manifeste aussi dans leur inclination respective à conclure des accords de libre-échange avec leurs voisins. Ces accords sont significatifs en ce qu'ils ressortent tout autant de logiques économiques - accroître les flux commerciaux, faciliter les investissements, etc. - que de logiques politiques. La conclusion d'un accord de libre-échange est en effet devenue un vecteur important de toute entreprise de rapprochement politico-diplomatique et cela, notamment pour la Chine. Ainsi, au terme de près de deux ans de négociations, la Chine a signé en novembre 2002 un accord pour la création d'une zone de libre-échange avec l'ASEAN en 2010. Projet fort ambitieux - à ce point ambitieux qu'il risque bien de connaître de sérieux obstacles cet accord n'en reste pas moins significatif sur le plan politique. Par-delà les stricts calculs économiques, il participe d'une stratégie globale visant à renforcer la position régionale prééminente de la Chine dans un climat de confiance et de coopération. Plus précisément, l'accord de libre-échange sino-ASEAN cherche à rassurer les petits pays d'Asie du Sud-Est en les convainquant que la forte croissance économique de la Chine représente une opportunité bien plus qu'une menace ${ }^{6}$. La Chine et l'ASEAN ont déjà entériné la première phase de leur projet général de partenariat économique, en concluant dès la fin 2004 un accord sur le commerce des biens, par lequel ils s'engagent à démanteler leurs barrières tarifaires et non tarifaires, et à instaurer un mécanisme de résolution des contentieux.

Ne perdant pas de vue l'activisme du voisin chinois en Asie du Sud-est, les Indiens ont tôt réagi... par voie d'imitation : dès novembre 2002, New Delhi a proposé à l'ASEAN la création d'une zone de libre-échange dans les dix années à venir, proposition au reste négociée en dix mois seulement. Ainsi New Delhi a-t-elle signé un accord pour la création future d'une zone de libre-échange avec l'ASEAN en octobre 2003, soit à peine un an après que la Chine eut conclu un accord similaire. New Delhi a clairement été soucieuse de faire montre de sa volonté politique et surtout de sa capacité bureaucratique à mettre en œuvre sa proposition initiale. L'activisme indien inclut par ailleurs des négociations de nature bilatérale. New Delhi a de fait signé un accord de libre-échange avec la Thaïlande fin 2003, et a achevé en juin 2005 les négociations pour un ambitieux accord de coopération économique générale avec Singapour, qui incorporera notamment une zone de libre-échange pour les biens et les services et un traité de promotion des investissements bilatéraux. Même si elle préfère l'option d'un accord multilatéral avec les dix membres de l'ASEAN, la Chine s'essaie également à des négociations bilatérales : elle a conclu un accord préférentiel avec la Thaïlande et conduit des négociations en ce sens avec Singapour.

11 En vertu de son activisme diplomatico-économique, l'Inde est parvenue à solidement s'arrimer au nouveau régionalisme asiatique. Le pays n'en continue pas moins de rester quelque peu à la marge de ce qui constitue le cœur de la régionalisation asiatique, notamment en termes d'interdépendances économiques. Une comparaison rapide des chiffres commerciaux et d'investissements révèle bien le poids relatif de l'Inde et de la Chine dans la région. Ainsi, pour l'année 2003, le volume commercial entre la Chine et l'ASEAN s'évaluait à près de 55 milliards de dollars. Il était en cela plus de quatre fois supérieur au volume commercial indo-ASEAN qui se limitait à quelque 12 milliards de 
dollars ${ }^{7}$. Surtout, le commerce intra-industries ${ }^{8}$ entre les économies de l'ASEAN et de la Chine est bien plus développé qu'il ne l'est avec l'Inde. Cette dernière n'a par exemple que récemment commencé à exporter des produits électroniques vers Singapour et la Malaisie.

De façon générale, la Chine s'avère bien plus intégrée à la régionalisation économique asiatique que l'Inde. En 2002, les économies d'Asie orientale représentaient environ $20 \%$ de l'ensemble du commerce extérieur de l'Inde alors qu'elles comptaient pour plus de $40 \%$ dans celui de la Chine. .'orientation des flux d'investissements est tout aussi révélatrice. Pour l'année 2002, les investissements directs en provenance des économies d'Asie orientale ne constituaient que $18 \%$ de l'ensemble des investissements étrangers annoncés en Inde ${ }^{10}$. Dans le cas chinois au contraire, les capitaux asiatiques représentaient une part essentielle de l'ensemble des investissements étrangers dans le pays (soit près $55 \%$ des investissements annoncés en 2002) avec, il est vrai, la région administrative spéciale de Hong Kong en tête de liste ${ }^{11}$. Certes, il ne faut pas oublier que les communautés chinoises d'outre-mer sont à l'origine d'une part très importante des flux de capitaux asiatiques à destination de la chine et que la diaspora indienne ne saurait en la matière jouer un rôle comparable. Mais, au-delà de ce paramètre, la plupart des nouvelles économies industrialisées d'Asie, telles Singapour ou la Corée du Sud, restent plus orientées vers la Chine que vers l'Inde. Quant au Japon, qui a historiquement joué un rôle moteur dans la régionalisation économique asiatique, il avait en 2002 investi cinq fois plus en Chine qu'en Inde ${ }^{12}$.

\section{Contribuer à la paix et à la sécurité dans la région}

13 Si l'Inde n'est pas aussi influente que la Chine en matière économique, elle bénéficie toutefois d'un meilleur capital de confiance dans le champ stratégique. En dépit d'une politique explicite de bon voisinage, s'accompagnant d'initiatives de rapprochement tous azimuts et d'efforts réitérés pour promouvoir des mécanismes de sécurité régionaux, le géant chinois ne semble toujours pas absout de son histoire trouble dans la région. Et si des progrès majeurs ont bien été accomplis en matière de restauration de la confiance et de socialisation régionale, les desseins futurs de Pékin ne laissent pas d'inquiéter. La modernisation militaire chinoise, de même que les nombreux différends territoriaux laissés irrésolus en mer de Chine expliquent la méfiance persistante de nombreux États d'Asie du Sud-Est, comme de ceux d'Asie du Nord-Est. Par comparaison, l'Inde fait figure de puissance relativement bénigne, et ne souffre pas de passif expansionniste ni même d'épisode conflictuel avec les États de la région, les divergences les plus graves étant dues à des facteurs désormais jugés extérieurs, c'està-dire aux alignements imposés par l'affrontement entre les deux Grands pendant la guerre froide. L'Inde a au reste largement exploité son image de puissance bienveillante pour se poser en pôle de stabilité sur la scène régionale asiatique, cela en contrepoint aux incertitudes entourant l'évolution de la Chine. Ainsi New Delhi entretient-elle des contacts militaires réguliers avec divers pays d'Asie orientale Singapour, le Vietnam, l'Indonésie et le Japon - contacts qui lui permettent entre autres choses d'affirmer une présence navale grandissante dans le golfe du Bengale, dans le détroit de Malacca et même en mer de Chine méridionale. développées. Et c'est fort prudemment que Pékin s'essaie à aborder certaines questions 
de sécurité régionale avec ses interlocuteurs en Asie du Sud-Est ${ }^{13}$. Premier pas encourageant, la Chine et l'ASEAN ont entrepris de coopérer en matière de lutte contre les menaces transnationales, dites «non traditionnelles», telles les narcotrafics. De même, en participant aux négociations pour la formalisation d'un Code de conduite en mer de Chine méridionale, la Chine a renoncé à l'usage de la force pour la résolution de ses différends dans cette zone. Pékin a par ailleurs accueilli avec intérêt l'idée de communauté de sécurité de l'ASEAN et a finalement accédé au Traité d'amitié et de coopération (TAC) de l'association, fin 2003. Or, entendant ne céder en rien à Pékin en matière de construction de la confiance en Asie du Sud-Est, l'Inde a tenu à accéder en même temps que la Chine au TAC, fin 2003. Dans le domaine stratégique, plus encore que dans n'importe quel autre domaine, Indiens et Chinois surveillent attentivement leurs avancées respectives.

\section{Regards croisés}

15 L'empressement commun de l'Inde et de la Chine à être partie prenante des processus d'intégration régionale conduit à s'interroger sur le regard que chacune de ces deux puissances porte sur le positionnement de l'autre en Asie. En d'autres termes, comment les acteurs chinois perçoivent-ils les efforts indiens pour s'arrimer à l'Asie orientale? Et inversement, comment les acteurs indiens intègrent-ils la Chine dans leur Look East policy?

\section{La Look East policy indienne vue de Chine}

Pour la communauté d'experts chinois s'intéressant à l'Inde, voire aux affaires asiatiques, la Look East policy est chose connue ${ }^{14}$. Plus précisément, cette communauté d'experts est très au fait des ambitions affichées, comme des avancées réelles de l'Inde en Asie du Sud-Est. Surtout, depuis le tout début des années 2000, leurs perceptions et analyses semblent avoir connu une évolution notable. Longtemps confinée au rang de puissance sud-asiatique, l'Inde est désormais reconnue comme un acteur destiné à exercer une influence par-delà le sous-continent, en particulier en Asie du Sud-Est, pour peu qu'elle soit capable de maintenir son haut taux de croissance économique. Il n'a ainsi pas échappé aux observateurs chinois que, si les voisins indiens souffraient d'un fort complexe d'infériorité pendant la décennie 1990, ils ont depuis peu regagné confiance et s'affirment comme une puissance ambitieuse. Loin d'être qualifiée de négative, cette évolution est au contraire bienvenue car elle est susceptible - juge-t-on - d'aider l'Inde à gérer son rapport à la Chine de façon plus mature.

17 La montée en puissance de l'Inde en Asie, notamment en Asie orientale est ainsi reconnue comme une tendance de fond, contre laquelle la Chine ne peut s'opposer, quand bien même elle le désirerait. Du point de vue chinois, la meilleure option pour gérer les ambitions indiennes reste de renforcer le climat de confiance et de coopération, de façon à désamorcer toute friction à venir. Cette politique de rapprochement pratiquée depuis la fin des années 1980 a connu l'une de ses avancées les plus significatives en juin 2003, lorsque le premier ministre A. B. Vajpayee et son homologue Wen Jiabao ont signé la première déclaration conjointe sino-indienne, par laquelle les deux voisins reconnaissent qu'ils ne présentent pas de menace l'un pour l'autre. En référence à leur politique asiatique respective, les deux parties ont même 
précisé qu'elles "voyaient de façon positive la participation de chacune dans les processus de coopération régionale et sous-régionale en Asie ${ }^{15}$, mention finalement assez vague mais qui vise probablement à démentir la thèse de leur rivalité sur le continent. De même, tout au long de sa visite en Inde, en avril 2005, le premier ministre Wen Jiabao a-t-il réitéré que les deux voisins étaient «des partenaires », et non " des rivaux ", et qu' « une Inde puissante et prospère ser[vai]t les intérêts de la Chine ${ }^{16}$.

Tout en reconnaissant certains des succès enregistrés par la Look East policy, la plupart des observateurs chinois se plaisent à souligner que Pékin entretient avec ses divers voisins d'Asie orientale des relations considérablement plus développées que l'Inde. Et en dépit de son nouveau dynamisme et de son immense potentiel, cette dernière ne peut encore se targuer d'avoir un poids économique comparable à la Chine. Le pays est par ailleurs perçu comme géographiquement excentré par rapport à la région, entendons par là qu'il n'a pas la position centrale et prééminente dont la Chine jouit. Finalement, la Chine admet la montée en puissance de l'Inde en Asie de façon d'autant plus positive qu'elle est elle-même convaincue de la prééminence et de la légitimité de son rôle régional et qu'elle mesure les nets progrès accomplis dans son rapport aux voisins d'Asie du Sud-Est ${ }^{17}$. À certains égards, c'est le succès de ses nouveaux concepts de politique extérieure qui expliquent la "sérénité relative » avec laquelle la Chine observe le développement de la Look East policy indienne. En d'autres termes, grâce à ses nouvelles orientations diplomatiques qui affichent une tonalité progressiste - «montée en puissance pacifique ", " nouveau concept de sécurité ", promotion du régionalisme et du multilatéralisme - la Chine estime entretenir des relations suffisamment étroites avec les pays membres de l'ASEAN pour ne pas se sentir menacée par le nouveau venu indien. À la désormais classique politique de bon voisinage, s'ajoute en effet le discours sur la «montée en puissance pacifique » que les nouveaux dirigeants chinois sont particulièrement enclins à promouvoir ${ }^{18}$. Cette thèse, qui avance que l'évolution de la Chine est bénéfique pour l'ensemble de l'Asie, implique en outre d'améliorer les relations avec l'Inde.

Pékin semble néanmoins soucieuse de s'assurer que l'Inde ne porte pas ombrage à sa prééminence en Asie orientale. Ainsi par exemple, la Chine n'a-t-elle pas particulièrement soutenu les tentatives indiennes pour élargir l'ASEAN +3 , organisation destinée de son point de vue aux pays d'Asie orientale seulement. Les réserves implicites de la Chine à l'égard des percées indiennes dans la région ont fini par transparaître au printemps 2005, à l'occasion des démêlés diplomatiques relatifs à l'organisation du premier Sommet de l'Asie orientale, censé se tenir à la fin de l'année ${ }^{19}$. Pékin, qui avait activement et officiellement promu la tenue d'un tel sommet, entendait bien réserver cet événement aux seuls membres de l'ASEAN+3. Or elle n'a guère été entendue en Asie du Sud-Est : redoutant sans nul doute la domination incontestée de la Chine à cette occasion, l'ASEAN a en effet décidé d'inviter l'Inde, conformément à son inclination traditionnelle à favoriser l'équilibre des puissances dans la région. Il n'aura ainsi pas échappé aux dirigeants chinois qu'en dépit de leurs efforts pour marginaliser l'Inde, cette dernière est de plus en plus incitée à se profiler comme l'alter ego de la Chine, sous l'influence même de l'ASEAN.

De la même façon, il serait trompeur de s'en tenir à une vision chinoise entièrement consensuelle à l'égard de la Look East policy indienne. Les questions stratégiques laissent ainsi entrevoir certaines nuances dans les perceptions chinoises. La communauté académique chinoise reconnaît par exemple porter un fort intérêt pour l'activisme 
naval de l'Inde (entendre par là, la modernisation navale indienne et la multiplication des exercices conjoints avec les autres puissances régionales). Cette même communauté académique souligne que cet intérêt ne se traduit pas nécessairement par de vives inquiétudes ou par un sentiment de rivalité inévitable dans les années à venir. Les experts chinois se félicitent d'ailleurs de voir leur pays lancer les premiers exercices navals conjoints avec des puissances étrangères - russes, pakistanaises et indiennes -, exercices d'ampleur limitée d'un strict point de vue technique, mais qui constituent une première en matière de construction de la confiance ${ }^{20}$.

Pourtant, les cercles militaires semblent nettement plus prudents à l'égard des avancées stratégiques indiennes en Asie du Sud-Est ${ }^{21}$. En premier lieu, il ne leur a pas échappé que l'Inde a été cooptée par l'ASEAN pour contribuer à l'équilibre des puissances dans la région, ce qui soulève à de nombreux égards l'hypothèse du contrepoids indien à la Chine. De plus, plusieurs experts chinois prennent note des rapides progrès accomplis par la politique de rapprochement indienne au Myanmar. Au point que certains d'entre eux ont désormais beau jeu de renverser l'argument indien sur la mainmise de la Chine au Myanmar et s'interrogent sur l'influence de New Delhi sur certains éléments de la junte militaire. Surtout, les cercles stratégiques chinois gardent un œil attentif sur les initiatives américaines en direction de l'Inde. Avant que les attentats du 11 septembre ne modifient les priorités stratégiques de Washington, Pékin s'est sérieusement inquiétée de la propension américaine à projeter l'Inde dans un rôle de contrepoids à la Chine ${ }^{22}$. Pour l'heure, une certaine confiance dans la tradition d'indépendance de l'Inde demeure. Il n'en reste pas moins que la communauté d'experts chinois - et à plus forte raison les cercles militaires - ont pris acte du fait que l'Inde figure désormais comme un paramètre dans la politique asiatique globale des États-Unis. Il est donc possible de voir la Look East policy indienne jouer en faveur des intérêts américains en Asie.

\section{La place de la Chine dans la Look East policy indienne}

Depuis la fin des années 1990, New Delhi a intégré et assimilé sur le plan diplomaticoéconomique l'importance critique des relations avec la Chine pour l'ensemble de sa politique asiatique. Il ne s'agit pas ici de suggérer que le rapport à la Chine était auparavant de rang secondaire, bien au contraire. Mais la Look East policy, telle qu'elle a été pratiquée pendant une grande partie de la décennie 1990, s'intéressait avant tout à l'Asie du Sud-Est, en plus du Japon et de la Corée du Sud, et était en quelque sorte dans un rapport tangent à la politique chinoise de l'Inde. Or, changement qualitatif s'il en est, vers la fin des années 1990, New Delhi a pleinement réintégré la Chine dans sa politique asiatique, au point que celle-ci est devenue un élément central de la Look East policy ${ }^{23}$.

Cette évolution résulte d'un changement de nature des relations bilatérales sinoindiennes: peu à peu émancipées des préoccupations purement stratégiques, notamment de celles qui portaient sur le différend frontalier datant de $1962^{24}$, elles se sont ouvertes aux logiques de coopération sur un large spectre, politique, économique, socioculturel et même militaire. Une certaine bonhomie prévaut désormais. En témoigne le changement de posture de Georges Fernandes, le ministre de la Défense du gouvernement de A. B. Vajpayee, qui avait dénoncé la menace chinoise peu avant les essais nucléaires de mai 1998 et qui, cinq ans plus tard, se rendait en visite d'amitié à 
Pékin pour promouvoir la coopération militaire bilatérale. Mais c'est surtout le déplacement du premier ministre A. B. Vajpayee en juin 2003 qui a envoyé un signal politique fort témoignant de la claire volonté de son pays à normaliser les relations avec Pékin. En s'engageant à accélérer les négociations sur la frontière, grâce notamment à la nomination de représentants spéciaux et dans une perspective politique, la partie indienne est revenue à une approche plus souple, infléchissant sa posture traditionnelle qui était jusqu'alors de revendiquer unilatéralement l'ensemble du territoire perdu en 1962. La visite de Wen Jiabao en avril 2005 a d'ailleurs permis aux deux voisins de signer un nouvel accord sur « les paramètres politiques et les principes directeurs pour un règlement de la question frontalière ", tout en réaffirmant «leur détermination à trouver une solution juste, raisonnable et mutuellement acceptable $\aleph^{25}$. Non seulement le contentieux frontalier ne constitue plus un obstacle majeur au développement des relations sino-indiennes, mais en plus les deux parties se sont engagées à trouver une solution communément satisfaisante dans les années à venir.

Enfin, ce qui initialement ressortait d'une initiative d'ordre avant tout symbolique - à savoir la promotion des liens économiques - prend peu à peu consistance. Les échanges commerciaux bilatéraux s'intensifient - ils sont passés de 3 milliards de dollars en 1999 à près de 14 milliards en 2004. La Chine est ainsi devenue le deuxième partenaire commercial de l'Inde après les États-Unis et les deux voisins envisagent désormais des négociations pour un accord bilatéral de libre-échange. L'effet d'annonce au niveau politico-diplomatique, c'est-à-dire le désir de confirmer sa volonté de coopération au plus haut du système décisionnel, reste un facteur important pour expliquer cette initiative qui, par ailleurs, effraie une grande partie des milieux industriels indiens. Mais il se double de préoccupations plus grandes, à tout le moins du côté indien : si le pays parvient à mettre en place des accords de libre-échange avec l'ASEAN d'une part, avec la Chine d'autre part, le concept de communauté économique asiatique s'en trouvera de facto renforcé ${ }^{26}$. Ce concept qui revient finalement à "ASEAN+3+Inde " permettrait à New Delhi de transcender le projet actuel de communauté économique d'Asie orientale qui la maintient dans un rapport périphérique. Cette approche consiste donc à reconnaître la centralité de la Chine au sein du régionalisme asiatique et à en jouer à son avantage pour mieux accroître l'intégration de l'Inde aux interdépendances économiques asiatiques.

Mais si l'Inde admet qu'une amélioration des liens avec la Chine aidera, entre autres choses, à renforcer son intégration au régionalisme asiatique, elle semble peu disposée à cautionner, encore moins à soutenir, les projets régionaux promus par Pékin. Une certaine réserve, voire un désagrément discret, se manifestent à l'égard des initiatives de coopération régionale dont la Chine voudrait être l'instigatrice. Ainsi du Forum de Boao pour l'Asie ${ }^{27}$ qui, dans un esprit panasiatique et paragouvernemental entend promouvoir les concertations économiques entre les pays du continent, sur le modèle du Forum économique mondial de Davos. Lors de chacune des sessions annuelles qui se sont tenues depuis le lancement du forum en 2001, New Delhi s'est bien gardée d'envoyer une représentation de premier plan $^{28}$. À dire vrai, aucun membre en exercice du gouvernement indien ne s'est jamais déplacé pour le forum. Par comparaison, New Delhi semble plus à son aise au sein de l'Asian Cooperation Dialogue, un projet tout aussi récent que le Forum de Boao, dont l'un des mérites principaux est sans doute d'avoir été promu par la Thaïlande, un "petit pays ", soutenant activement la Look East policy indienne qui plus est ${ }^{29}$. 
CARTE 1

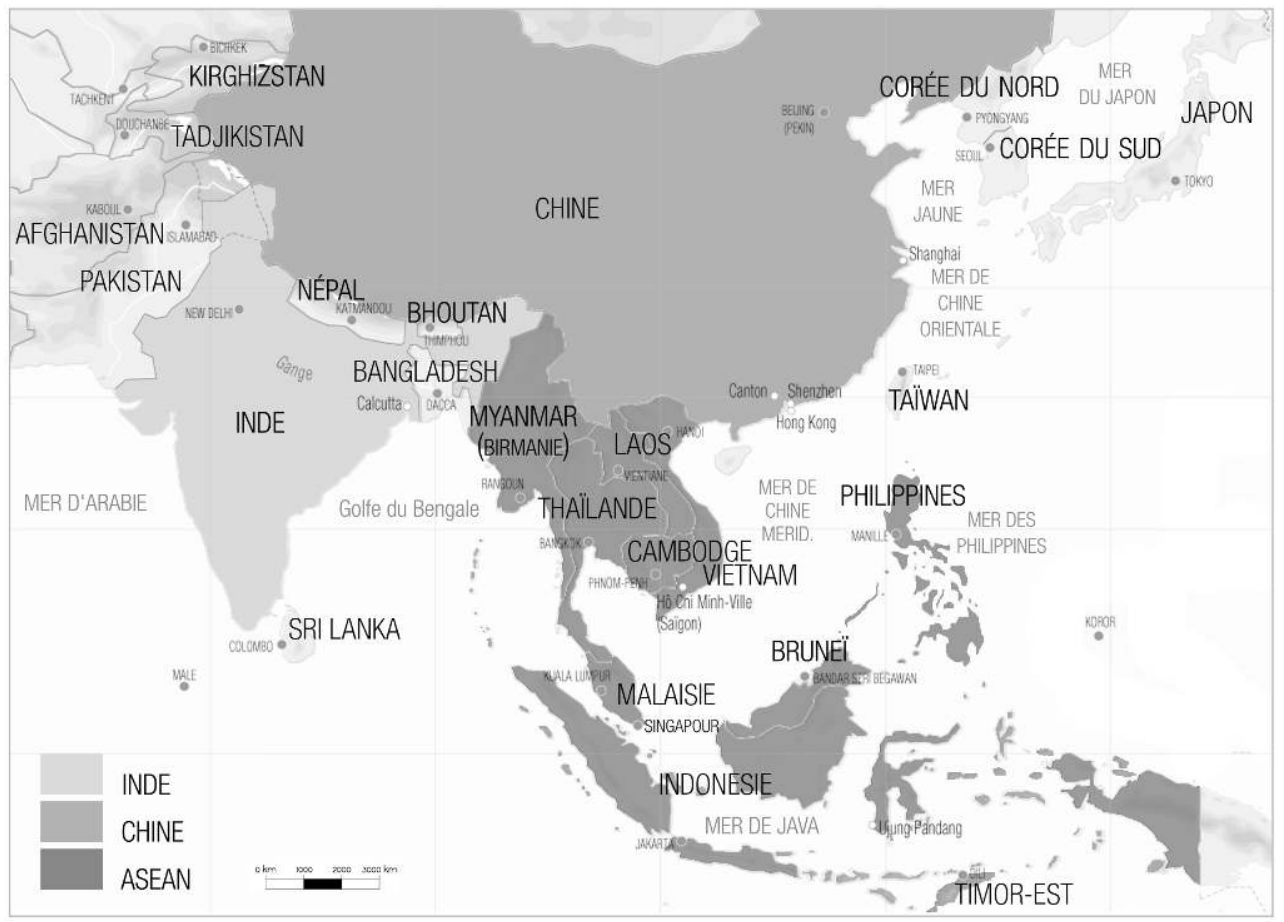

INDE, CHINE ET ASEAN

\section{CARTE 2a}

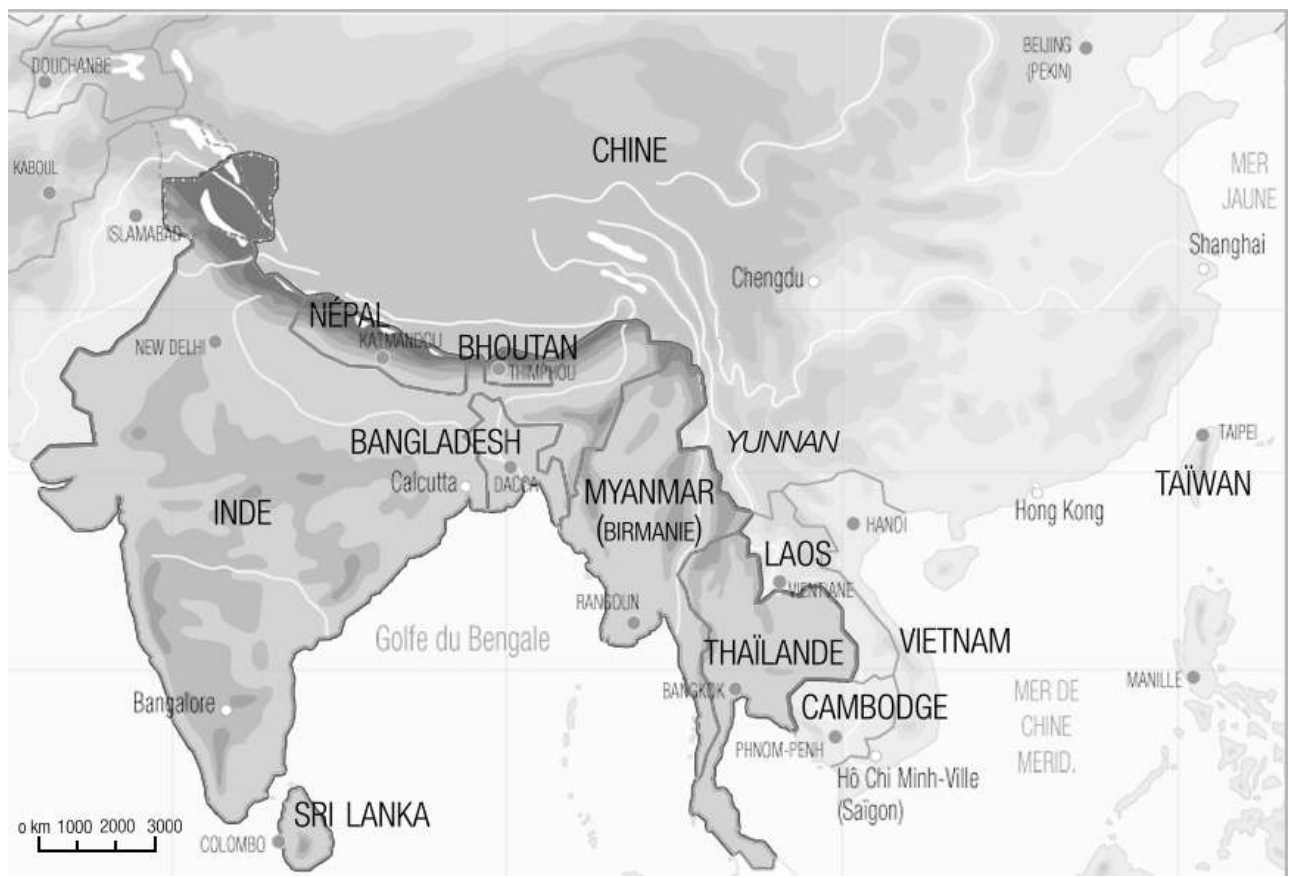

BIMSTEC 
CARTE 2b

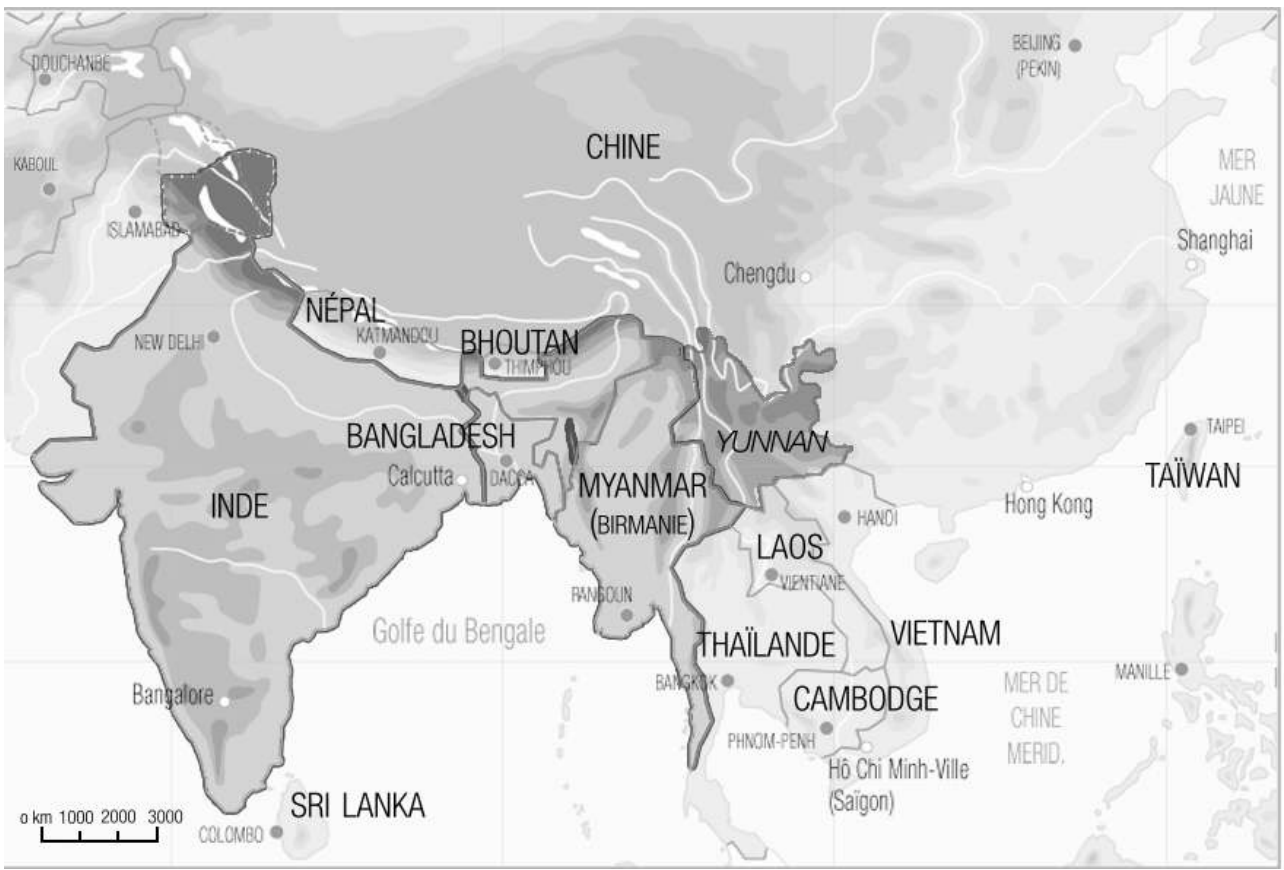

Initiative de Kunming

\section{CARTE 2C}

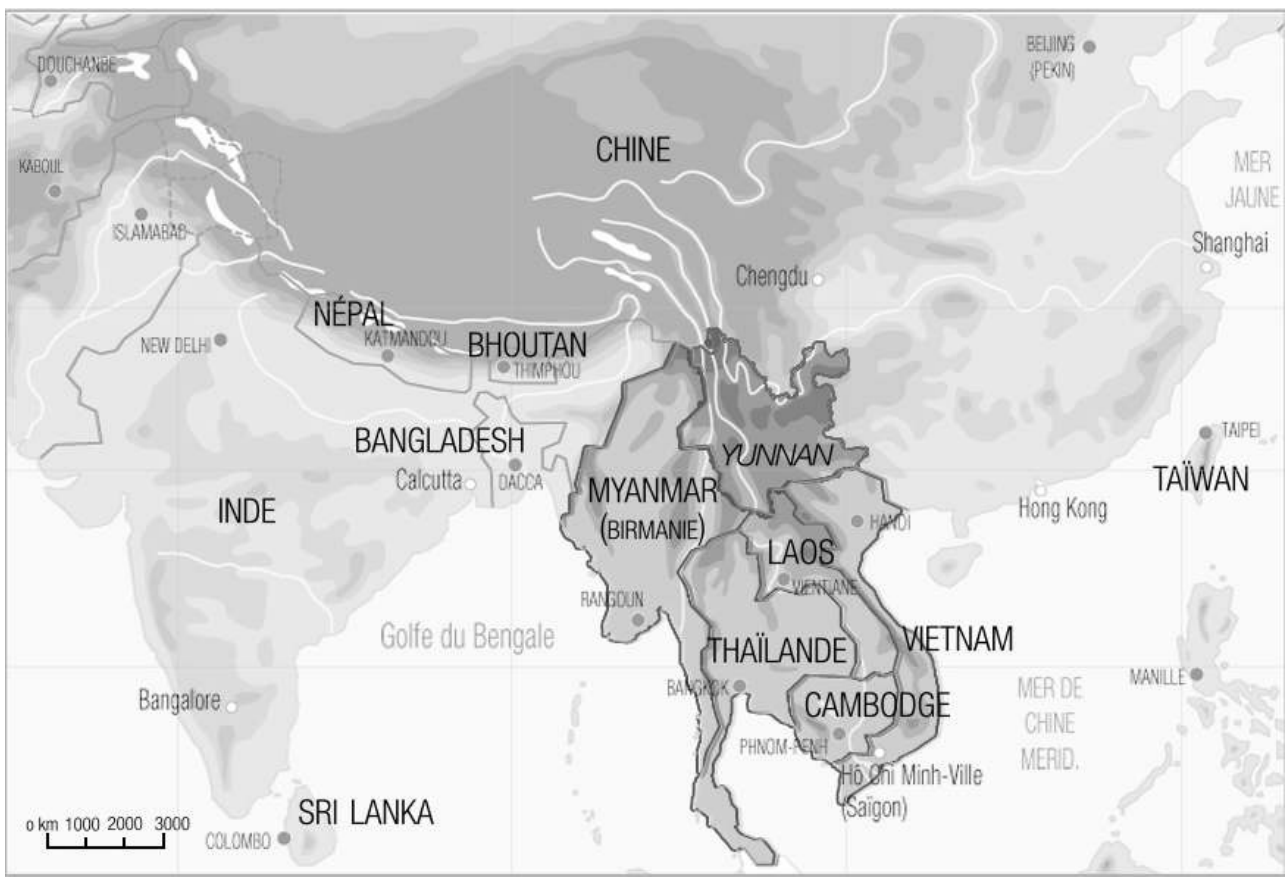

Coopération Grand-Mékong 


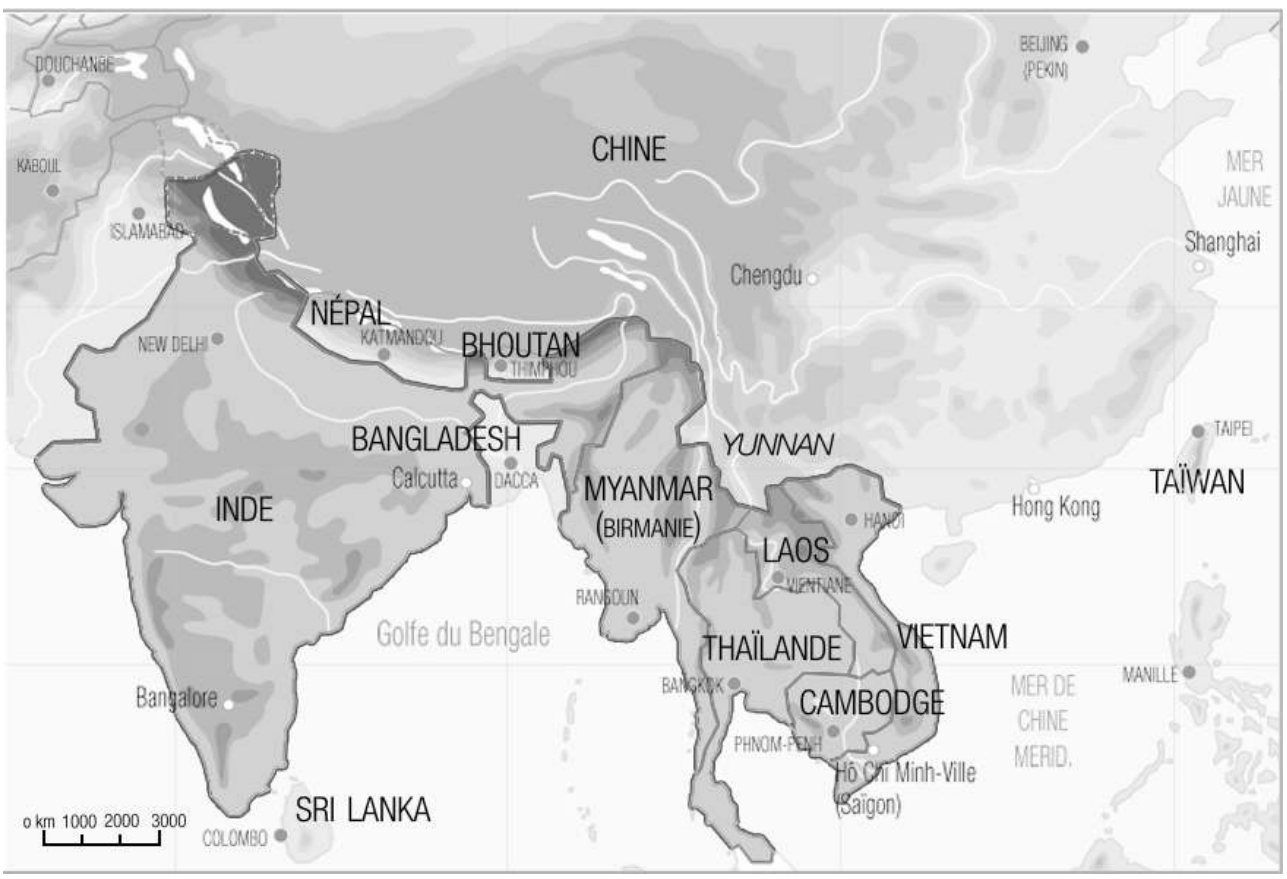

Coopération Mékong-Gange

C'est aussi cette même réserve à l'égard des initiatives régionales chinoises qui explique en partie la stagnation de l'Initiative de Kunming, un projet de coopération sousrégional tout à fait novateur entre le Nord-Est de l'Inde, le Myanmar, le Bangladesh et le Sud-Ouest de la Chine. Promue par la province du Yunnan, et fortement soutenue par Pékin en cela, cette initiative, appelée aussi BCIM (pour Bangladesh, Chine, Inde, Myanmar), ne parvient pour l'instant pas à surmonter le scepticisme d'une bonne partie de la communauté stratégique indienne. Car, en plus d'être perçue comme un projet sous leadership chinois, l'Initiative de Kunming touche à des préoccupations relatives à la sécurité et à la stabilité du Nord-Est, une région où New Delhi craint toute interférence extérieure. De plus, l'Inde aime autant promouvoir des projets de coopération régionale excluant la Chine. À ce titre, sa préférence pour le BIMSTEC ${ }^{30}$, qui se superpose géographiquement à l'Initiative de Kunming mais présente l'avantage d'exclure le Yunnan pour mieux se recentrer sur le littoral du golfe du Bengale, est révélatrice. De la même façon, New Delhi s'essaie à effectuer quelques percées dans la région du Mékong en se gardant bien de conjuguer ses efforts avec ceux de la Chine. Cette dernière est pourtant déjà fort engagée dans cette sous-région. La province du Yunnan participe en effet au programme de développement du "Grand Mékong " (GMS), qui inclut le Cambodge, le Laos, le Myanmar, la Thaïlande et le Vietnam. New Delhi a de son côté lancé en juillet 2000, le projet de Coopération Mékong-Gange (MGC) qui l'associe à cinq pays de l'ASEAN riverains du fleuve Mékong: la Thaïlande, le Myanmar, le Vietnam, le Laos et le Cambodge. Or ce programme embryonnaire s'est créé sans la participation de la Chine qui a pourtant demandé à y être associée.

Il faut finalement bien reconnaître que la Look East policy participe d'une vision ambitieuse du rôle et de l'influence de l'Inde en Asie et qu'à ce titre, elle entretient une certaine ambivalence à l'égard de la Chine. Cette ambivalence transparaît bien en matière stratégique où certaines suspicions ont la vie dure, notamment lorsqu'elles ont 
trait aux activités chinoises au Pakistan et au Myanmar. Mais à bien des égards, ces mêmes craintes aident à légitimer une politique de modernisation militaire menée à marche forcée. La doctrine navale indienne rendue publique en juin 2004 résume bien le mélange d'inquiétudes et d'ambitions que suscite le rapport à la Chine ${ }^{31}$. Selon la doctrine, la mission future de la marine indienne est en effet d'assurer une capacité de dissuasion conventionnelle et nucléaire à l'encontre des États de la région capables d'agir contre les intérêts sécuritaires de l'Inde, à savoir : le Pakistan et la Chine. Les auteurs de la doctrine jugent en effet que les projets chinois d'acquérir des groupes de combats aéroportés affectent directement les intérêts futurs de l'Inde, et recommandent donc l'achat de sous-marins nucléaires. Ces observations qui viennent quelque peu à l'encontre de la bonhomie ambiante ont le mérite de rappeler que les relations entre deux puissances ascendantes comme l'Inde et la Chine ne sauraient exclure certains éléments de compétition. Ce faisant, toute la problématique des ajustements et repositionnements en cours se trouve précisément dans l'aptitude de leurs dirigeants à gérer ces éléments de tensions latents.

\section{Conclusion}

La comparaison des approches respectives de l'Inde et de la Chine face au nouveau régionalisme asiatique et surtout, l'analyse des regards croisés que chacun des deux pays porte l'un sur l'autre montrent que la thèse de la rivalité sino-indienne en Asie n'est pas une donnée inévitable. Pékin et New Delhi sont toutes deux en phase de repositionnement sur la scène régionale asiatique. Le premier, déjà prédominant en Asie, a particulièrement à cœur de rassurer l'ensemble de son voisinage et de projeter une image de géant affable et responsable. La seconde, en quête d'un statut de puissance majeure en Asie, exprime ses nouvelles ambitions de façon d'autant plus active et extravertie qu'elle sait sa marginalité par rapport à une région qui se concentre avant tout sur la Chine. Or se sont précisément ces dynamiques de repositionnements à l'échelle régionale qui portent Indiens et Chinois à certains réajustements significatifs dans leurs relations bilatérales. La logique même de sa politique de bon voisinage ou de "montée en puissance pacifique " conduit Pékin à apporter un soin particulier à son rapport à l'Inde, l'un de ses plus importants voisins, l'un des rares par ailleurs avec qui le différend sur la frontière terrestre n'a toujours pas été normalisé. Le désir indien de prendre pleinement part au nouveau régionalisme asiatique pose comme condition nécessaire et indispensable non seulement de pacifier le rapport à la Chine, mais d'en jouer pour s'intégrer plus avant à la région.

Nous ne saurions bien sûr ignorer que les politiques de conciliation de la Chine comme de l'Inde obéissent avant tout à un pragmatisme dicté par les circonstances présentes, qui ne les empêche en rien de chercher à accroître leur influence sur le long terme, en Asie orientale, mais aussi dans l'océan Indien et en Asie Centrale. Il n'en reste pas moins que le rapport sino-indien à l'échelle bilatérale comme régionale est avant tout affaire de volonté politique au plus haut de l'appareil décisionnel des deux États. C'est tout le sens qu'il faut donner au ballet diplomatique auquel se livrent les dirigeants chinois et indiens et aux grandes déclarations qui accompagnent désormais chacun de leurs déplacements. Ainsi la visite du premier ministre Wen Jiabao en avril $2005 \mathrm{a}$-t-elle été l'occasion de conclure un "partenariat stratégique et de coopération pour la paix et la prospérité ", censé ancrer plus fermement encore l'amitié sino-indienne, tout en 
marquant l'entrée des relations bilatérales dans une nouvelle phase qui intéressera, non pas seulement l'Asie, mais le monde dans son ensemble. Notons cependant que, plus encore que la nouvelle déclaration de partenariat stratégique, c'est le commentaire de Wen à propos de la requête de New Delhi pour l'attribution d'un siège au Conseil de sécurité qui a été relevé par les hôtes indiens : il laissait entendre qu'il y était favorable, dans la mesure où la Chine "comprenait et soutenait pleinement l'aspiration indienne à jouer un rôle encore plus important dans les affaires internationales et aux Nations unies $»^{32}$. Si cette déclaration quelque peu ambiguë et manifestement destinée à flatter les interlocuteurs indiens annonce un infléchissement réel de la position chinoise, elle aura signalé une avancée décisive dans les relations entre les deux géants.

\section{BIBLIOGRAPHIE}

- ACHARYA A., « Seeking Security in the Dragon's Shadow: China and Southeast Asia in the Emerging Asian Order ", Institute of Defence and Strategic Studies Working paper $n^{\circ} 44$ (Singapour), March 2003.

- COMMISSION ÉCONOMIQUe ET SOCIALE POUR L'ASIE ET LE PACIFIQUE, Annuaire Statistique pour l'Asie et le Pacifique 2003, New York, Nations unies, 2004.

• CHERIAN J., « Moving closer », Frontline, vol. 22, n 9, April 23-May 6, 2005.

- FOOT R., « China’s Regional Activism: Leadership, Leverage and Protection », Global Change, Peace and Security, vol. 17, n², June 2005, pp. 141-153.

- GARVER J. W., « The China-India-U.S. Triangle: Strategic Relations in the Post-Cold War Era », NBR Analysis, vol. 13, n 5, October 2002 [http://www.nbr.orgl.

- HAN H., « Friends or Foes: Mutual Perception between two Asian Giants », in I. SAINT-MÉZARD et J. K. CHIN (éds), China and India: political and strategic perspectives, Hong Kong, CAS, 2005.

• HU J., « China's development is an opportunity for Asia », Xinhua, 23 April 2004.

- INTEGRATED HEADQUARTERS, MINISTRY OF DEFENSE (Navy), Indian Maritime Doctrine, INBR 8, New Delhi, MoD, April 2004.

- LIU F. et P. RÉGNIER (éds.), Regionalism in East Asia: Paradigm Shifting? London, Routledge Curzon, 2003.

- MINISTRY OF COMMERCE AND INDUSTRY, SIA Newsletter, January 2003 [http://dipp.nic.in].

- MINISTRY OF COMMERCE OF THE PRC, 2003 Report on Foreign Investment in China [http:// english.mofcom.gov.cn/].

• MOHAN R. C., « Looking East: Phase Two », The Hindu, 11 April 2002.

- NAGESH K. (éd.), Towards an Asian Economic Community: Vision of a New Asia, New Delhi, RIS, 2004. 
- PANG Z., « The impact of China-ASEAN Free Trade Agreement on Regional Trade », The Journal of East Asian Affairs, vol. XVIII, n² 2, Fall-Winter 2004, pp. 311-339.

- SIDHU W. P. S. et J. YUAN, China and India: Cooperation or Conflict, Boulder Colo., Lynne Rienner, 2003.

- STUBBS R., « ASEAN Plus Three: Emerging East Asian Regionalism? », Asian Survey, vol. 42, n 3, May-June 2002, pp. 440-455.

- TANG S. et ZHANG Y., "China's Regional Strategy: an Interpretation », Centre of Regional Security Studies (CRSS) Working Paper (Chinese Academy of Social Sciences, Beijing), n 1, March 2004, pp. 1-22.

- WALKER M., « Walker's world: Battles around new Asia summit », The Washington Times, 2 April 2004.

- WEN J., « Main points of Wen's speech at summit of ASEAN plus China, Japan and ROK », People's Daily online, 30 November 2004.

- XU J., « A peaceful Rise: China's Strategic Option », International Studies (China Institute of International Studies, Beijing) (Vol. 2, March 2004), pp. 1-20.

- " Active India Role in China-based Boao Forum Urged by Former Premier », PTI news agency 3 November 2003.

- «China's position paper on the new security concept », 6 August 2002 [http:// www.fmprc.gov.cn].

- «Chinese and Indian navies take to the water together », China Daily, 14 November 2003.

- Declaration on Principles for Relations and Comprehensive Cooperation between the Republic of India and the People's Republic of China, 23 June 2003 [http://meaindia.nic.in/].

- «Indo-ASEAN relationship should lead to an Asian Economic Community: PM inaugurates India ASEAN business meet ", Prime Minister's Office, 19 October 2004.

-Joint statement of the Republic of India and the People's Republic of China, 11 April 2005 [http:// meaindia.nic.in/].

\section{NOTES}

1. Le refus d'intégrer l'Inde à l'APEC au long des années 1990 reposait sur l'argument selon lequel les réformes économiques progressaient à un rythme trop lent et hésitant. Dans le cas de l'ASEM, l'Inde a été exclue parce que la représentation asiatique de l'organisation a préféré se limiter aux pays d'Asie orientale seulement. En cela, l'ASEM a été le précurseur de l'ASEAN+3.

2. F. Liu et P. Régnier (éds), Regionalism in East Asia : Paradigm Shifting ? London, Routledge Curzon, 2003; R. Stubbs, «ASEAN Plus Three: Emerging East Asian Regionalism? », Asian Survey, vol. 42, $\mathrm{n}^{\circ}$ 3, May-June 2002, pp. 440-455.

3. Voir notamment le discours du premier ministre Wen Jiabao au sommet de l'ASEAN +3 de 2004, en ce qu'il est fort emblématique de l'activisme chinois: "Main Points of Wen's Speech at Summit of ASEAN plus China, Japan and ROK », People's Daily online, 30 November 2004.

4. R. Foot, "China's Regional Activism: Leadership, Leverage and Protection ", Global Change, Peace and Security, vol. 17, n² 2, June 2005, pp. 141-153.

5. Le partenariat entre l'Inde et l'ASEAN touche surtout à la coopération économique, et à la lutte contre le terrorisme et la criminalité internationale. 
6. Z. Pang, «The Impact of China-ASEAN Free Trade Agreement on Regional Trade ", The Journal of East Asian Affairs, vol. XVIII, n 2, Fall-Winter 2004, pp. 311-339.

7. Chiffres disponibles sur le site officiel du secrétariat de l'ASEAN : http://www.aseansec.org.

8. Commerce constitué d'échanges croisés de produits d'une même branche industrielle.

9. Chiffres compilés à partir des données disponibles dans : Commission économique et sociale pour l'Asie et le Pacifique, Annuaire Statistique pour l'Asie et le Pacifique 2003, New York, Nations unies, 2004, pp. 126-128 et pp. 211-213. Il est à noter que ces chiffres n'incluent pas Taiwan.

10. Ministry of Commerce and Industry, SIA Newsletter, January 2003, accessible à l'adresse: http://dipp.nic.in/.

11. Ministry of Commerce of the PRC, 2003 Report on Foreign Investment in China, p. 64. Rapport accessible sur le site officiel $d u$ ministère du Commerce de la RPC: http:// english.mofcom.gov.cn/.

12. Données accessibles sur le site du ministère des Finances du Japon : http://www.mof.jp.

13. A. Acharya, "Seeking Security in the Dragon's Shadow: China and Southeast Asia in the Emerging Asian Order ", Institute of Defence and Strategic Studies Working paper $n^{\circ} 44$ (Singapour), March 2003.

14. Ce point est dérivé d'une série d'entretiens menés en juin 2004 avec divers experts chinois associés aux principaux centres de recherche sur l'Asie du Sud à Pékin, à savoir le China Institute for International Relations, le China Institute for Contemporary International Relations, la School of International Studies (Peking University) et l'Institute of Asia-Pacific Studies (Chinese Academy of Social Sciences).

15. Declaration on Principles for Relations and Comprehensive Cooperation between the Republic of India and the People's Republic of China, 23 June 2003, accessible à l'adresse: http:// www.meaindia.nic.in/.

16. J. Cherian, « Moving Closer », Frontline, vol. 22, n 9, April 23-May 6, 2005.

17. S. Tang et Y.Zhang, "China's Regional Strategy: an Interpretation ", Centre of Regional Security Studies (CRSS) Working Paper (Chinese Academy of Social Sciences, Beijing), $\mathrm{n}^{\circ} 1$, March 2004, pp. 1-22.

18. Voir le discours du président $\mathrm{Hu}$ Jintao lors de la cérémonie d'ouverture de la session $2004 \mathrm{du}$ Forum Boao pour l'Asie: « China's development is an opportunity for Asia », Xinhua, 23 April 2004, et: J. Xu, «A Peaceful Rise: China's Strategic Option", International Studies (China Institute of International Studies, Beijing), vol.2, March 2004, pp.1-20. Pour le «nouveau concept de sécurité », voir : «China's position paper on the new security concept », 6 August 2002, accessible sur le site officiel du ministère des Affaires étrangères chinois : http://www.fmprc.gov.cn.

19. M. Walker, «Walker's World: Battles around New Asia Summit», The Washington Times, 2 April 2004.

20. "Chinese and Indian navies take to the water together », China Daily, 14 November 2003.

21. Certaines des inquiétudes chinoises sont bien documentées dans l'ouvrage de W. P. S. Sidhu et J. Yuan, China and India: Cooperation or Conflict, Boulder Colo., Lynne Rienner, 2003. Elles s'exprimaient aussi de façon plus perceptible dans les entretiens avec les chercheurs du CICIR, institut proche des cercles de défense.

22. J. W. Garver, "The China-India-U.S. Triangle: Strategic Relations in the Post-Cold War Era ", NBR Analysis, vol. 13, n5, October 2002, accessible sur le site: http://www.nbr.org; Han Hua, «Friends or Foes: Mutual Perception between two Asian Giants », in I. Saint-Mézard et J. K. Chin (éds), China and India: Political and Strategic Perspectives, Hong Kong, CAS, 2005.

23. R. C. Mohan, « Looking East: Phase Two ", The Hindu, 11 April 2002

24. Une courte guerre, traumatisante pour New Delhi, avait opposé en 1962 l'Inde à la Chine, qui avait franchi largement la Ligne Mac Mahon, frontière contestée entre le Tibet et le Nord-est indien. La question n'est toujours pas réglée aujourd'hui, non plus que le contentieux portant sur les frontières de l'Himalaya oriental. 
25. Joint Statement of the Republic of India and the People's Republic of China, 11 April 2005. Accessible à l'adresse: http://meaindia.nic.in/.

26. K. Nagesh (éd.), Towards an Asian Economic Community: Vision of a New Asia, New Delhi, RIS, 2004, 203 p.; "Indo-ASEAN relationship should lead to an Asian Economic Community: PM inaugurates India ASEAN business meet », Prime Minister's Office, 19 October 2004.

27. Le Forum tire son nom de la ville de Boao, dans l'île tropicale de Dongyu, dans la province du Hainan.

28. Les membres indiens les plus actifs au sein du Boao sont la FICCI, une chambre patronale indienne, et I. K. Gujral, qui a participé à la session 2003, mais en qualité d'ancien premier ministre. Voir les remarques de I. K. Gujral sur les inhibitions indiennes à propos du forum Boao : « Active India Role in China-based Boao Forum Urged by Former Premier », PTI news agency, New Delhi, 3 November 2003.

29. Ainsi, lors de la session 2004 qui, pour l'occasion, se tenait à Qingdao en Chine, New Delhi n'a pas hésité à dépêcher son nouveau ministre des Affaires étrangères, Natwar Singh.

30. L'acronyme BIMSTEC signifie: Bangladesh, India, Myanmar, Sri Lanka, Thailand Economic Cooperation. Après l'arrivée du Népal et du Bhoutan, l'acronyme a changé de sens en 2004, pour devenir: the Bay of Bengal Intiniative for Multisectoral Technical and Economic Cooperation. On l'appelle parfois aussi ВОВСОМ, pour Bay of Bengal Community.

31. Integrated Headquarters, Ministry of Defense (Navy), Indian Maritime Doctrine, INBR 8, New Delhi, MoD, April 2004, 148 p.

32. Joint statement of the Republic of India and the People's Republic of China, 11 April 2005, accessible à l'adresse: http://meaindia.nic.in/.

\section{RÉSUMÉS}

La nouvelle vague de régionalisme qui caractérise l'Asie depuis la crise financière de 1997 révèle l'influence grandissante de deux acteurs majeurs, la Chine bien sûr, dont la prééminence sur le voisinage s'est affirmée au cours des dernières années, mais aussi - et de plus en plus - l'Inde. Chinois et Indiens rivalisent d'initiatives pour être partie prenante des nouvelles dynamiques d'intégration à l'œuvre en Asie orientale. Parallèlement, une analyse croisée des perceptions qu'Indiens et Chinois ont de leurs avancées respectives en Asie orientale, éclaire comment la Chine et l'Inde, qui ont eu des relations troubles par le passé, se positionnent l'une par rapport à l'autre dans leur course à l'intégration régionale.

The new wave of regionalism that has characterised Asia since the 1997 financial crisis reveals the growing influence of two major players : China, clearly, whose predominance in the area has been confirmed in the past few years, and - increasingly - India. Both are competing to participate in the new dynamics of integration at work in East Asia. A cross-analysis of Indian and Chinese perceptions of their respective inroads in East Asia highlights how these two powers, wich in the past have experienced uneasy relations, position themselves in relation to each the other in the race towards regional inegration. 
INDEX

Index géographique: Inde, Chine

Keywords : India, China, regionalism, East Asia, ASEAN+3

Mots-clés : régionalisme, Asie orientale, ASEAN+3

\section{AUTEUR}

\section{ISABELLE SAINT-MÉZARD}

Chercheur associée au Centre of Asian Studies de l'université de Hong Kong. Isabelle Saint-Mézard est docteur en relations internationales de l'Institut d'études politiques de Paris, sa thèse portant sur la Look East policy indienne dans les années 1990. Elle a été affiliée au Centre de sciences humaines de New Delhi. Ses recherches actuelles portent sur les relations extérieures de l'Inde, en particulier sur les relations sino-indiennes, ainsi que sur le régionalisme asiatique. Chercheur associée au Centre of Asian Studies de l'université de Hong Kong, elle est coéditrice avec James K. Chin d'un ouvrage collectif intitulé : China and India : Political and Strategic Perspectives (Centre of Asian Studies, Hong Kong University, 2005).

isabellesaintmezard@yahoo.fr 